\title{
Damage Accumulation under Repeated Reverse Stressing of Sn-Ag Solder Joints
}

\author{
K.C. CHEN, ${ }^{1}$ A. TELANG ${ }^{2}$ J.G. LEE ${ }^{2}$ and K.N. SUBRAMANIAN ${ }^{2,3}$ \\ 1.-Materials Engineering Department, California Polytechnic State University, San Luis Obispo, \\ CA 93407. 2.-Department of Chemical Engineering and Materials Science, Michigan State \\ University, East Lansing, MI 48824-1226. 3._E-mail: subraman@egr.msu.edu
}

\begin{abstract}
To better understand the effect of repeated reverse stress in solder joints, a new testing method was developed. Tin-silver solder joints were fabricated, constrained between $\mathrm{Cu}$ blocks, and then subjected to repeated shear loading in a tensile tester. Constant strain amplitudes were applied to simulate service conditions. However, large loads were used to accelerate the damage accumulation. Microstructural features of the damage were very similar to those found with studies on thermomechanical fatigue (TMF) of small, single shear lap samples. Concentrated-shear banding or striations were observed to form along Sn dendrites. The load behavior of the solder with each cycle and during hold times at the extreme strain amplitude was consistent with damage accumulating with each successive cycle. Effects of strain amplitude, hold times at the stress extremes, number of cycles, and solder-joint thickness were found to play significant roles on the stress-strain behavior and surface damage.
\end{abstract}

Key words: Stress-strain behavior, Sn-Ag, solder joints

\section{INTRODUCTION}

Solder joints encounter repeated reversed stressing during their service. Such an encounter is not only due to traditional mechanical loading, such as that caused by engine vibrations or sudden impact in automotive and aerospace applications, but also due to thermal fluctuations. ${ }^{1}$ However, in the sedentary office computer type of applications, the latter seems to be the predominant cause of solder-joint failures. During service, several phenomena that take place in the solder affect the joint's reliability. Some of these factors are aging, ${ }^{2-6}$ microstructure evolution, ${ }^{7}$ creep $^{8-11}$ and stress relaxation, ${ }^{12}$ and deformation under reversed-stress states (because of the coefficient of thermal expansion (CTE) mismatches between the solder and substrates during thermal excursions, or mechanical vibrations and impact loading). ${ }^{13}$

Although the reliability of the solder joint is influenced by virtually all these phenomena, the relative importance of each of these factors and their influence on each other is not clearly understood. Accelerated tests with actual solder joints containing the attached components do not provide any means to understand the true influence of each of these phenomena separately. ${ }^{14,15} \mathrm{As}$ a result, most of the fundamental studies are carried out with solder joints with simple geometries. 8 ,9 There have been focused studies on lead-free, Sn-based solders to understand individual phenomenon, such as aging, ${ }^{2,3,4,6,16}$ low- and hightemperature creep,${ }^{17-20}$ stress relaxation, ${ }^{12}$ microstructural evolution, ${ }^{13}$ surface-damage accumulation caused by thermomechanical fatigue (TMF), ${ }^{21,22}$ and residual-mechanical properties after TMF. ${ }^{23}$

The main purpose of this study is to evaluate the behavior of eutectic Sn-Ag solder joints under isothermal-reversed stressing in conjunction with stress relaxation at the stress extremes. The stressstrain behavior and surface-damage accumulation in eutectic $\mathrm{Sn}-\mathrm{Ag}$ solder joints are investigated. While addressing only one part of a much larger and complicated scenario, the results from this study will assist in the overall understanding of solder joints.

\section{EXPERIMENTAL PROCEDURES}

Although actual solder joints experience stress reversals during service, their configurations are not suitable for imposing stress reversals by external means. To alleviate this problem, a large solder sam- 
ple geometry was designed and is pictured in Fig. 1. Three $\mathrm{Cu}$ blocks, $3 / 8$ in. $\times 3 / 8$ in. $\times 1$ in., were attached together by two symmetrical Sn-Ag solder joints in a Y-like configuration. Predrilled holes in the $\mathrm{Cu}$ blocks allowed the entire specimen to be loaded into the Instron tensile tester, as depicted in Fig. 2. Samples were loaded in tension, and subsequently, the solder joints experienced pure shear states. In addition, this particular sample geometry and test setup allowed easy reversal of the shear stress (by using compression mode) without unloading the sample. Previous studies ${ }^{24}$ required the sample to be completely unloaded, removed, and flipped over to apply reverse shear. In realistic service conditions, the solder would be prevented from fully relaxing to a zero load before being subjected to reversed shear. ${ }^{12}$ Thus, this new test setup allows the study of damage accumulation and mechanical behavior of constrained solder joints under isothermal, repeated reverse stressing along with stress relaxation at extreme strains.

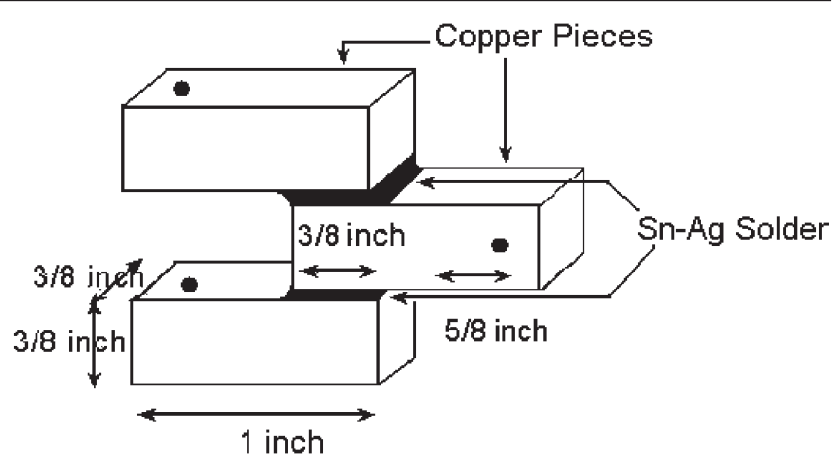

Fig. 1. Sample geometry for repeated reverse shear experiments.

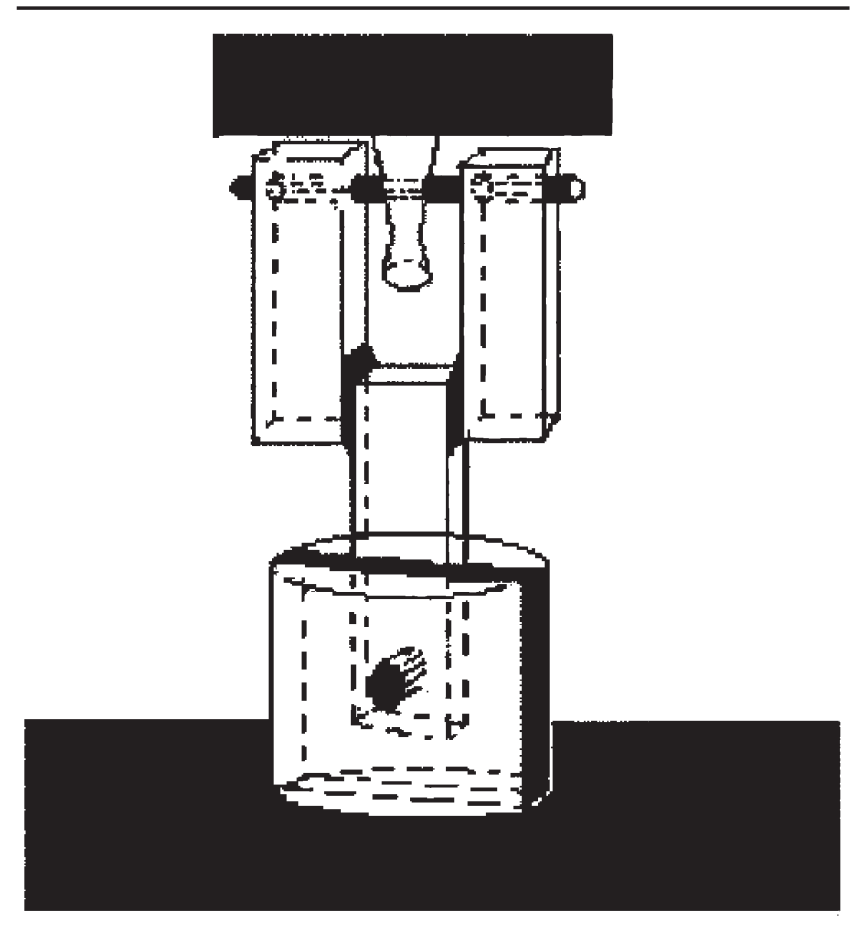

Fig. 2. Constrained solder samples loaded in an Instron tester for cycling between minimum and maximum shear-strain amplitudes.
The samples were prepared in similar fashion to small, single shear lap samples used in TMF studies. ${ }^{22}$ Copper blocks were polished, cleaned with $\mathrm{HNO}_{3}$, and washed with methanol. Steel pins were placed through the predrilled holes to align the $\mathrm{Cu}$ pieces during joining. Eutectic Sn-Ag solder paste was then applied to designated $3 / 8$ in. $\times 3 / 8$ in. areas on the $\mathrm{Cu}$. The amount of solder used controlled the thickness of the solder joint, and the two sides of solder in the sample were made to be of the same thickness as close as possible. A range of thickness $(300-900 \mu \mathrm{m})$ was employed to study the effect of joint thickness on the mechanical behavior of the solder joints. Steel blocks were also used to help align the $\mathrm{Cu}$ blocks and to restrict movement during the joining operation. The entire assembly sat on an aluminum plate and was heated until melting of the solder occurred (roughly after $10 \mathrm{~min}$ ). The plate was then transferred to a larger aluminum block for cooling to room temperature (for $15 \mathrm{~min}$ ). The solder joints were then polished for microstructural evaluation.

The test specimens were tested in shear at room temperature with an Instron tensile tester at a displacement rate of $0.5 \mathrm{~mm} / \mathrm{min}$. The samples were first loaded in tension to a predetermined strain to avoid any mechanical slack during testing. Different hold times at the extreme strains were employed (for stress-relaxation studies) before applying shear in the reverse direction by using the compression mode of the tester. Thus, cycling between a maximum shear strain and minimum shear strain was performed (Fig. 3). Load-displacement data was collected during the stressing of the samples, while load-time data was obtained during hold times at strain extremes.

To gauge any machine effects that may be incurred with these studies, preliminary tests were performed on a solid $\mathrm{Cu}$ sample of the same geometry but without any solder joints. An initial drop in load at the maximum strain (in tension mode) of $<10 \mathrm{~N}$ was consistently found within the first $5 \mathrm{sec}$, despite the actual load value. During the hold time (or stress-relaxation mode), the load was very steady after $5 \mathrm{sec}$. On the other hand, hold times in compression mode (or at the minimum strain) was found to always result in a very small increase in load, again despite the actual load value. Thus, stress-relaxation studies were delegated to hold times at the maximum strain amplitude only (i.e.,

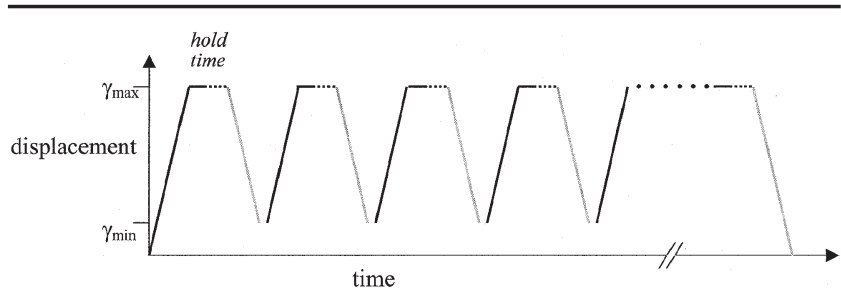

Fig. 3. Displacement control was used to achieve reverse shear by cycling between minimum and maximum shear-strain amplitudes without removal of samples. 
tension mode). Any changes greater than $10 \mathrm{~N}$ in the load during hold times or in the peak load after cycling could be attributed to the solder material.

Microstructures of the solder joints were analyzed with light microscopy and scanning electron microscopy (SEM). The progression of damage accumulation was documented on a few samples by unloading after some cycles, recording the microstructure, and then repeating the steps after more loading cycles. Earlier studies ${ }^{25}$ concluded that the solder microstructure did not change over a few days time after being unloaded for analysis.

\section{RESULTS AND DISCUSSION}

\section{Microstructures: Damage Accumulation}

Because of the large shear-strain amplitudes applied to the samples, damage quickly accumulated after a few cycles, in contrast to the thousands of cycles experienced by the TMF samples with similar damage features. Figure 4 depicts periodic striations that arose after 20 cycles (with a maximum strain amplitude of 0.75). The striations appear at a specific angle to the interface, and two sets of parallel striations occurred in this particular sample, shown in Fig. 4. Typically, the samples had only one set of striations within a solder joint. The striations are regularly spaced at roughly $20 \mu \mathrm{m}$.

Upon closer examination (Fig. 5), the striations appeared to form along the Sn-rich dendrites. The SEM (Fig. 6) definitively shows the striations between the interdendritic regions with $\mathrm{Ag}_{3} \mathrm{Sn}$ particles. The pattern is very similar to results from eutectic solder-fatigue studies with cantilever bending. ${ }^{1}$ Striations were reported to be decorated with $\mathrm{Ag}_{3} \mathrm{Sn}$ particles. The striations seem to develop as localized banding because of repeated shearing cycles. Thus, the microstructure appears to play a significant role in the damage accumulation and mechanical behavior of the Sn-Ag solder joints.

Because of the heat flow during the solidification portion of the joining process, long Sn-rich dendrites form across the thickness of the joint. The primary dendrites are parallel and regularly spaced roughly $20 \mu \mathrm{m}$ apart throughout most of the solder joint. The dendrites often make an angle to the interface. Secondary dendrite arms are apparent and are surrounded by tiny $\mathrm{Ag}_{3} \mathrm{Sn}$ particles.

A sample that experienced 20 cycles of 0.75 strain was carefully polished to reveal the underlying microstructure and indeed shows dendrites underneath the striation and banding, as shown in Fig. 7. Given the extreme shear strain applied to the solder material, deformation of the Sn-rich phases is to be expected. The yield stress of pure $\mathrm{Sn}$ is $11 \mathrm{MPa}$, and the long, connected regions of Sn allow the damage to be localized along the dendrite arms. In TMF samples, damage occurs by grain-boundary decohesion and sliding between Sn grains. ${ }^{12,22}$

A different sample of similar thickness was tested under similar strain amplitudes but was unloaded

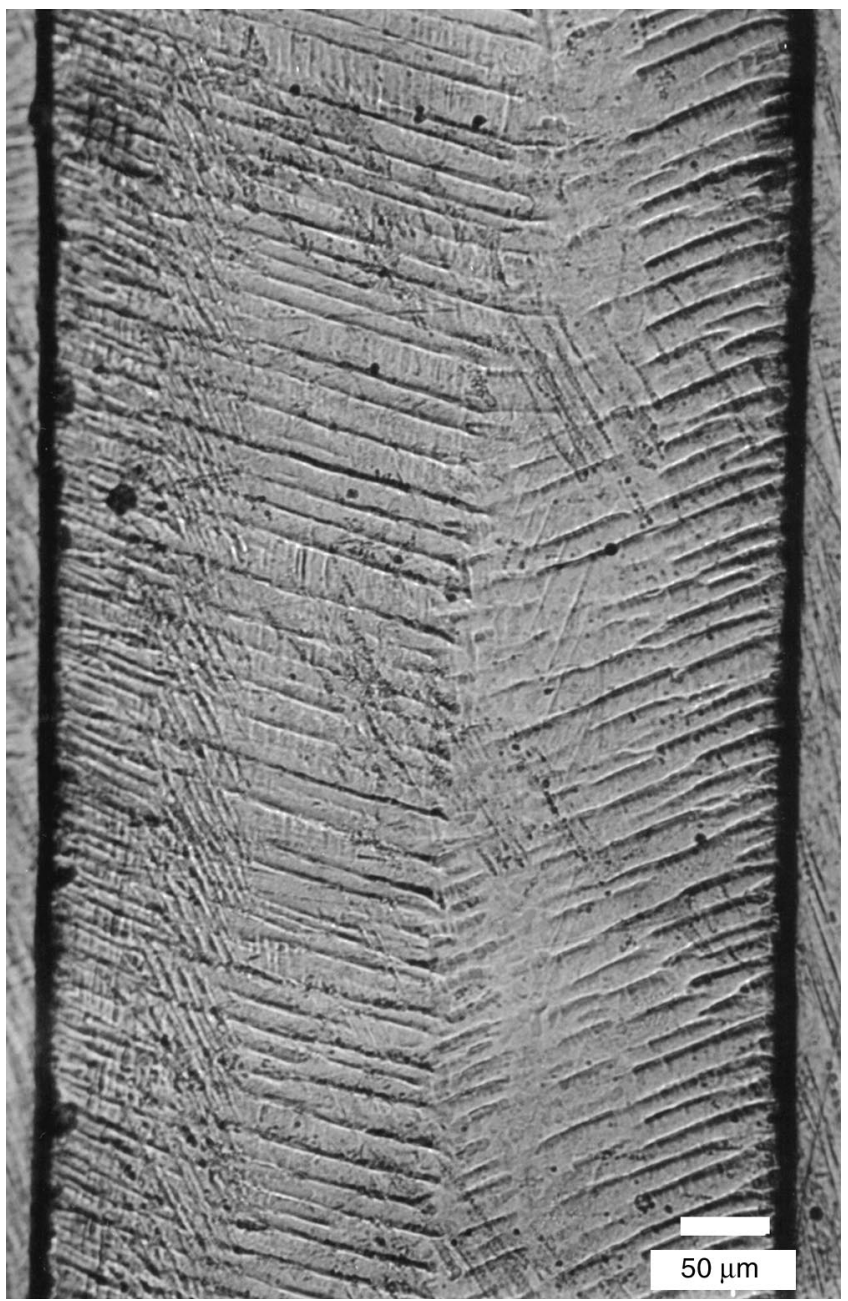

Fig. 4. Parallel striations formed in eutectic Sn-Ag solder of $712-\mu \mathrm{m}$ thickness after 20 cycles to a maximum strain amplitude of 0.75 (and load $\sim 3.5 \mathrm{kN}$ )

and analyzed after 1 cycle, 4 cycles, and 15 cycles. The initiation and progression of damage can be observed in Fig. 8. Damage is accumulated with successive cycles of reverse shearing. After the first cycle, the striations appear along some of the Sn dendrites near the interface. More striations appear with successive cycles throughout the sample. In addition, existing striations deepen and grow with repeated cycles. After large numbers of cycles or large applied strains, the accumulated damage can be severe enough to obscure the periodic striations and can resemble large shear bands that have been documented in other studies. ${ }^{1,22}$

However, large enough loads had to be encountered to produce visible damage in the solder joints. Samples that were subjected to roughly $2.5 \mathrm{kN}$ or more displayed the characteristic striations visible by light microscopy (Table I). Taking into consideration the two solder areas, the threshold load represents a stress level of $13.7 \mathrm{MPa}$, which is greater than the yield stress of pure $\mathrm{Sn}$. The load value was found to be the most defining parameter to show damage. Joint thickness and strain amplitude were 


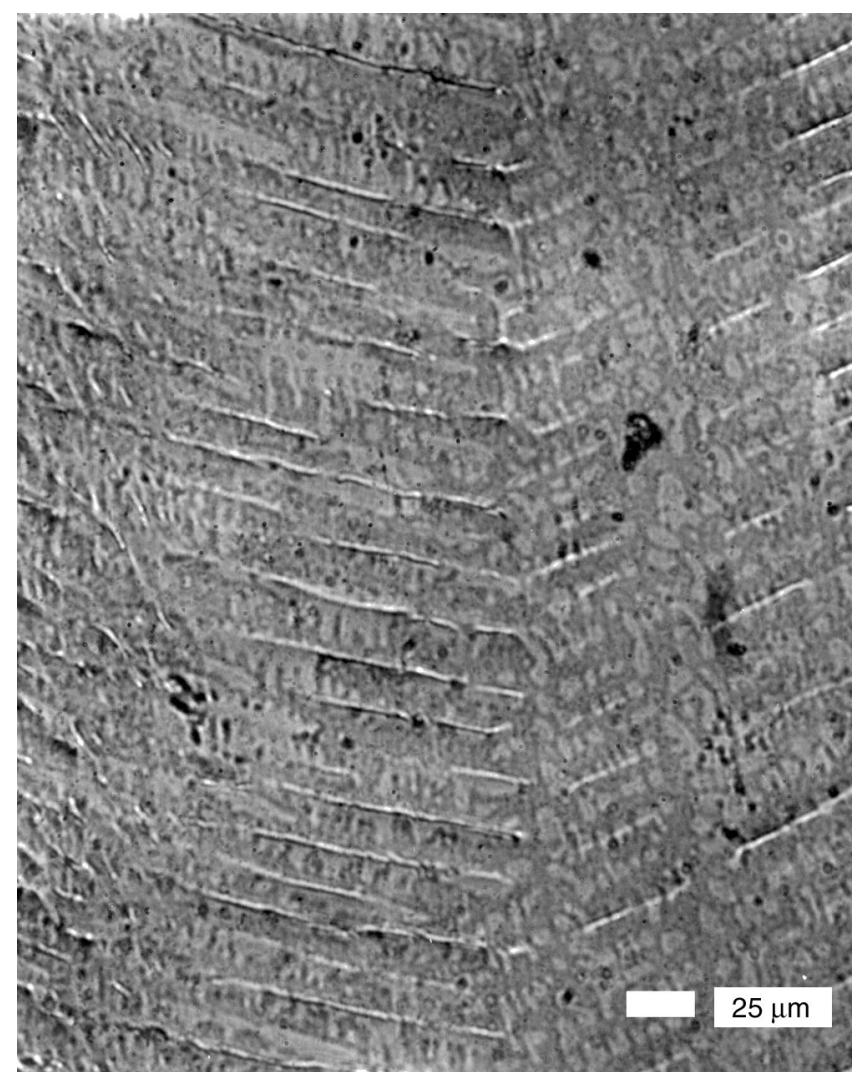

Fig. 5. Striations in the same directions and spacings as Sn dendrites of the eutectic solders of sample in Fig. 4.

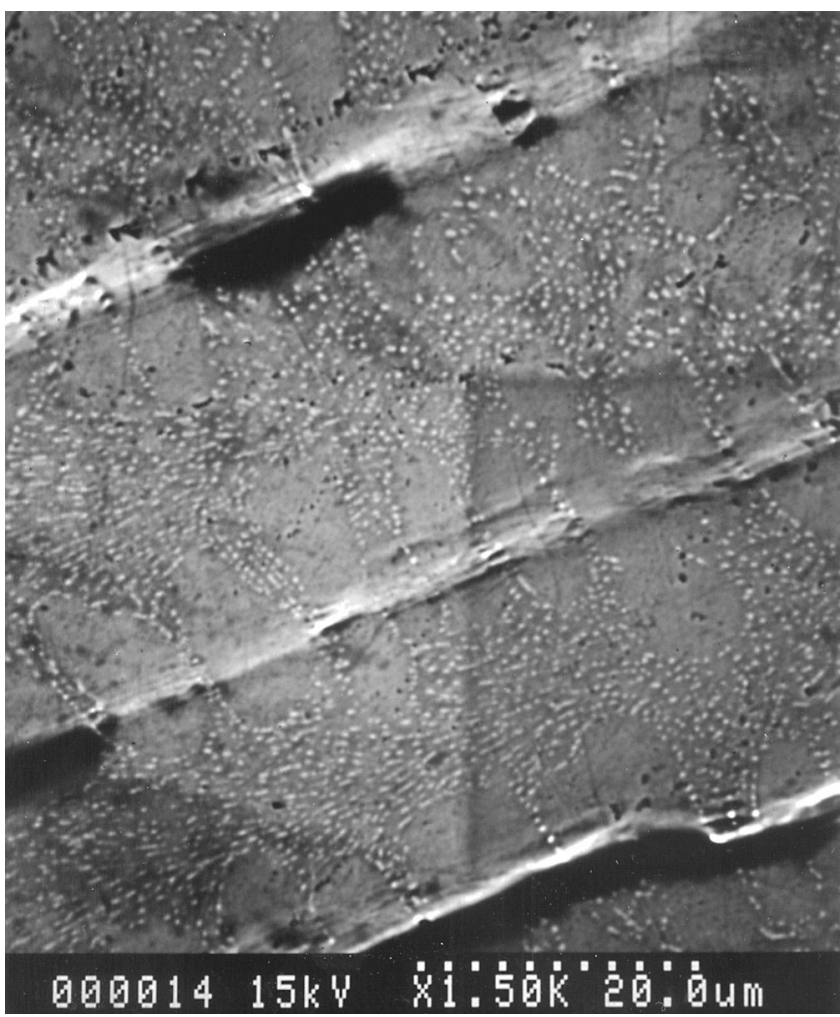

Fig. 6. SEM image of striations along Sn dendrites and between $\mathrm{Ag}_{3} \mathrm{Sn}$ particles.

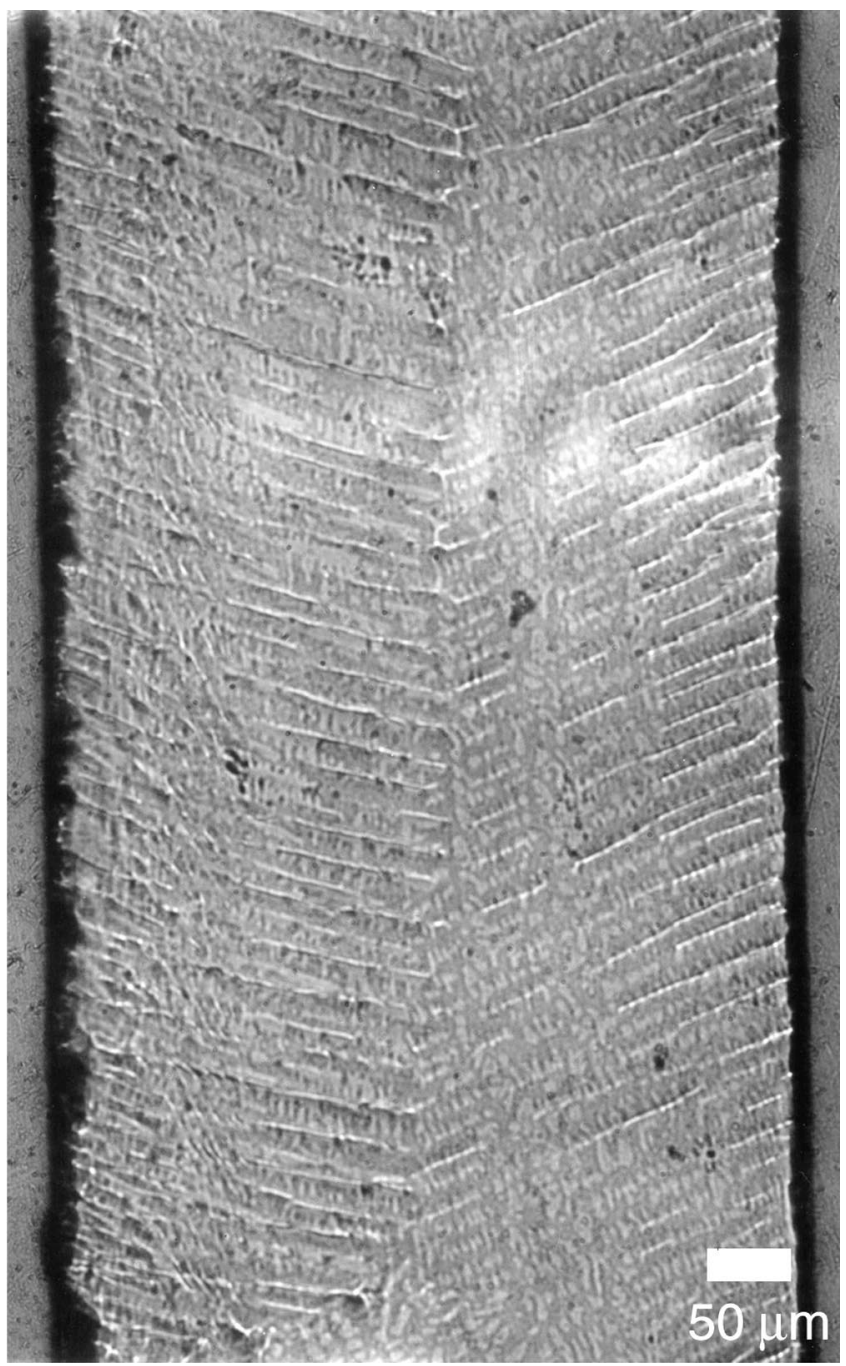

Fig. 7. The 712- $\mu \mathrm{m}$ sample (Fig. 4) polished down to reveal the underlying microstructure of the Sn dendrites along the concentrated shear banding or striations.

pertinent factors only in that they affected the amount of load applied to the samples.

In TMF samples, the damage is primarily shear banding and grain-boundary sliding/decohesion. The strain per cycle is much smaller, about 0.002 . However, damage starts to appear within $100 \mathrm{TMF}$ cycles of thermal excursions between $15^{\circ} \mathrm{C}$ and $150^{\circ} \mathrm{C}$. If strain per thermal cycles accumulates in an additive fashion, $100 \mathrm{TMF}$ cycles will result in a strain of 0.2. The TMF results in surface damage at low shear-strain values caused by high single-crystal anisotropy of Sn grains in regards to the CTE and Young's modulus and caused by the existence of high-temperature effects associated with the TMF cycles. While the damage from TMF appears on the surface of the solder samples, the isothermal mechanical stress reversals seem to accommodate deformation within the joint body, as well as on the free surfaces.

Although the samples (and, thus, their microstructures) used in this study are much larger than those of realistic joints, the identified mi- 


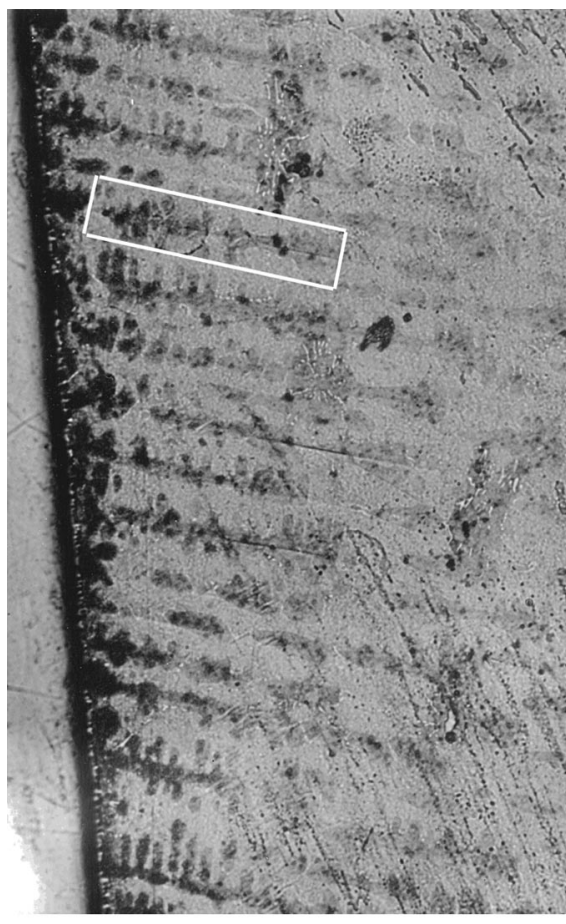

a
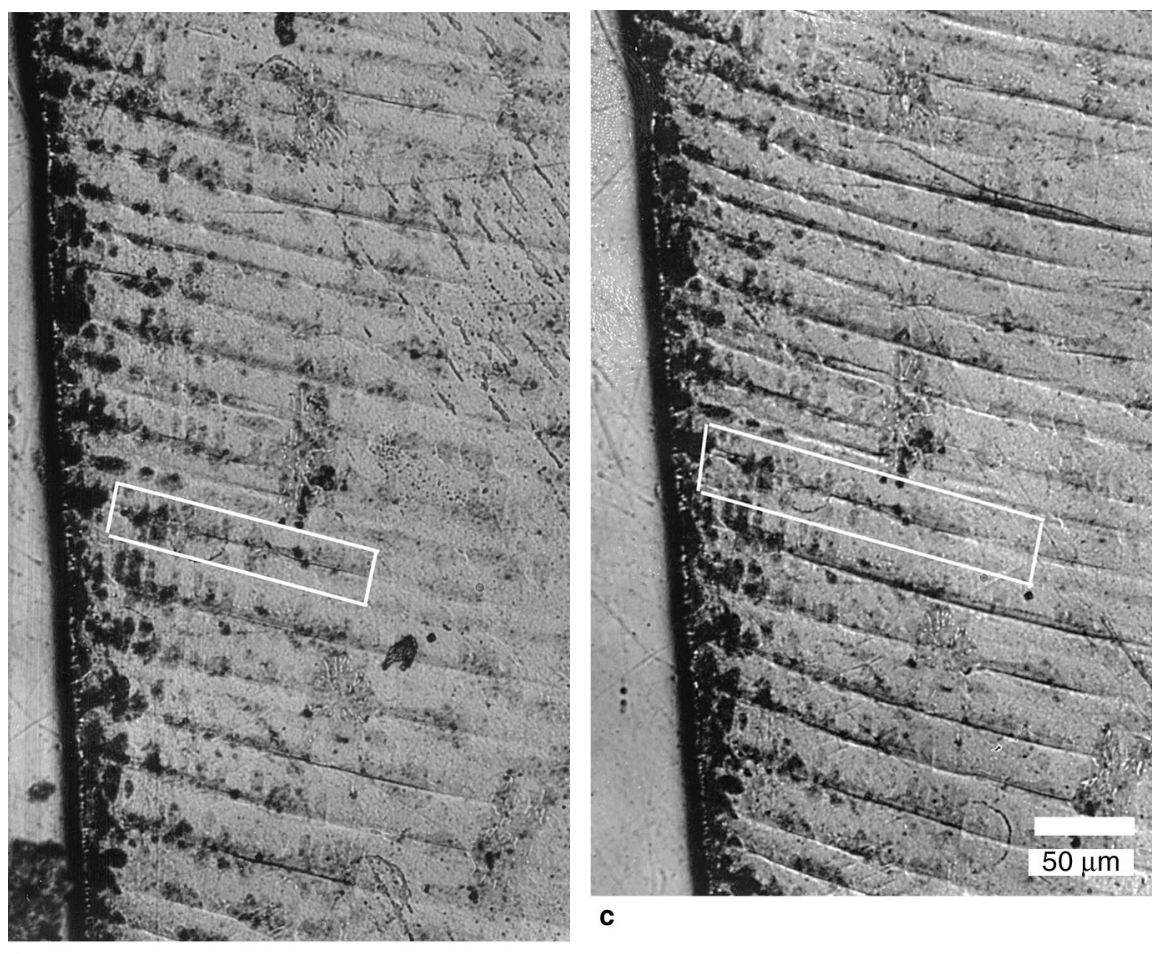

c

Fig. 8. Solder joint of 700- $\mu \mathrm{m}$ thickness subjected to $3.0 \mathrm{kN}$ or 0.7 strain after (a) 1 cycle, (b) 4 cycles, and (c) 15 cycles. Striations along Sn dendrites become more intense with additional cycles of reverse shearing.

Table I. The Applied Strain Amplitude, Maximum Load, Hold Time at $\gamma_{\max }$, Number of Cycles, and Observation of Surface Damage for Samples Used in This Study

\begin{tabular}{|c|c|c|c|c|c|}
\hline Sample Thickness & Strain & Maximum Load (kN) & Hold Time (min) & Cycles & Damage? \\
\hline $331 \mu \mathrm{m}$ & 0.75 & 1.37 & 0.5 & 15 & no \\
\hline $309 \mu \mathrm{m}$ & 1.0 & 1.59 & 0.3 & 4 & no \\
\hline $333 \mu \mathrm{m}$ & 1.5 & 3.09 & 0.5 & 5 & yes \\
\hline $389 \mu \mathrm{m}$ & 1.5 & 4.3 & 5 & 7 & yes \\
\hline $460 \mu \mathrm{m}$ & 0.5 & 1.0 & 5 & 8 & no \\
\hline $455 \mu \mathrm{m}$ & 0.75 & 1.60 & $14 \mathrm{~h}$ & 1 & no \\
\hline $509 \mu \mathrm{m}$ & 0.75 & 1.97 & 1 & 20 & no \\
\hline $508 \mu \mathrm{m}$ & 0.75 & 2.37 & 5 & 15 & some \\
\hline $500 \mu \mathrm{m}$ & 1.0 & 3.6 & 5 & 15 & yes \\
\hline $700 \mu \mathrm{m}$ & 0.70 & 3.04 & 0.5 & 1 & little \\
\hline $712 \mu \mathrm{m}$ & 0.75 & 3.5 & 5 & 20 & yes \\
\hline $979 \mu \mathrm{m}$ & 0.5 & 3.2 & 5 & 15 & yes \\
\hline $934 \mu \mathrm{m}$ & 0.75 & 4.5 & 0.5 & 10 & yes \\
\hline
\end{tabular}

crostructural effect on solder damage can help with the development of joining techniques to achieve better properties. Isolation of the Sn grains would avoid the concentrated banding or striations and may result in longer fatigue life. Modification of the microstructure through rapid solidification or fabrication of composite structures may also yield better properties.

\section{Reverse-Shear Mechanical Behavior}

Results from current tests correlate well with the proposed mechanisms for damage accumulation. During the stress-relaxation portion of the tests, the stress drops as a function of time, while strain (or displacement) is held constant (Fig. 9). The magnitude of these load drops is consistently the largest for the very first cycle. Given a high enough load, striations form, and crack initiation occurs with the first cycle (Table I).

The magnitude of load (or stress) drops during hold times at maximum strain decreases with successive cycles and eventually appears to have the same decrease in load per cycle (Fig. 9). In addition, the peak load of the next cycle decreases with successive cycles. Damage accumulates with the cycling between strain amplitudes, and the solder cannot withstand as much load as before. After several cycles, stress relaxation slows down dramatically. 


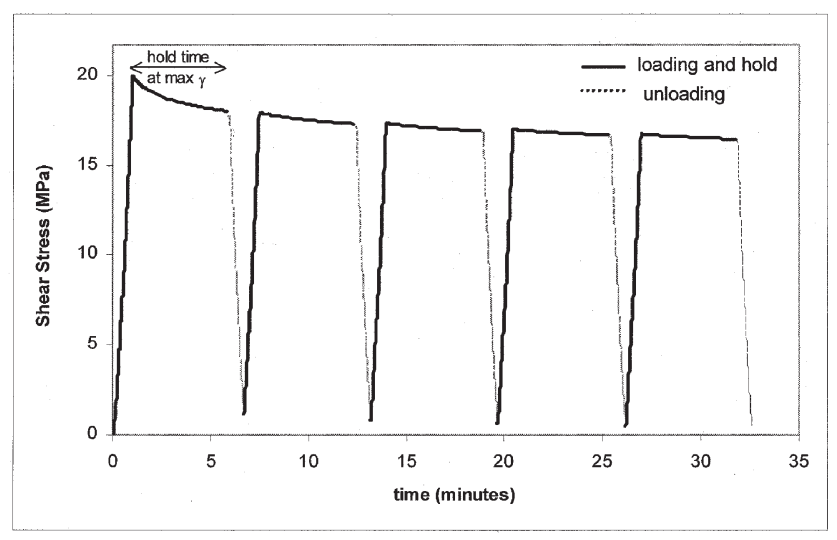

Fig. 9. Stress versus time plot of repeated, reverse shear tests for 5 cycles. Loading, holding at maximum strain amplitude (i.e., stress relaxation), and unloading portions of tests are shown.

The effect of hold times at the maximum strain amplitude was also investigated. One sample was loaded to $\sim 1.6 \mathrm{kN}$ for a strain of 0.75 and held for roughly $12 \mathrm{~h}$. Figure 10 shows that the load approaches approximately $95 \%$ of the original peak load after roughly $5 \mathrm{~h}$ and reveals that full relaxation (to zero load) never occurs within the time frame of the experiment. Physical constraints imposed upon the solder joints result in a behavior significantly different from that of bulk-solder samples.

A different sample (of similar thickness) was held at a maximum strain amplitude of 0.75 (and $\sim 1.96$ $\mathrm{kN}$ ) for only 1 min but was cycled 20 times (Fig. 11). (The plot displays only the stress-relaxation data and does not show the loading or reverse loading portions.) The load also drops rapidly at the beginning of the stress-relaxation period. The peak load for the next cycle is much lower, but it reaches the load experienced at the end of the first hold period. Each following cycle has a lower peak load and shows smaller load-drop magnitudes per cycle than the first cycle. The load drops to about $97 \%$ of the original peak load after about 6 cycles (or 10 min of testing), and the load drop per cycle at later stages are relatively small.

Comparison of Figs. 10 and 11 clearly shows that

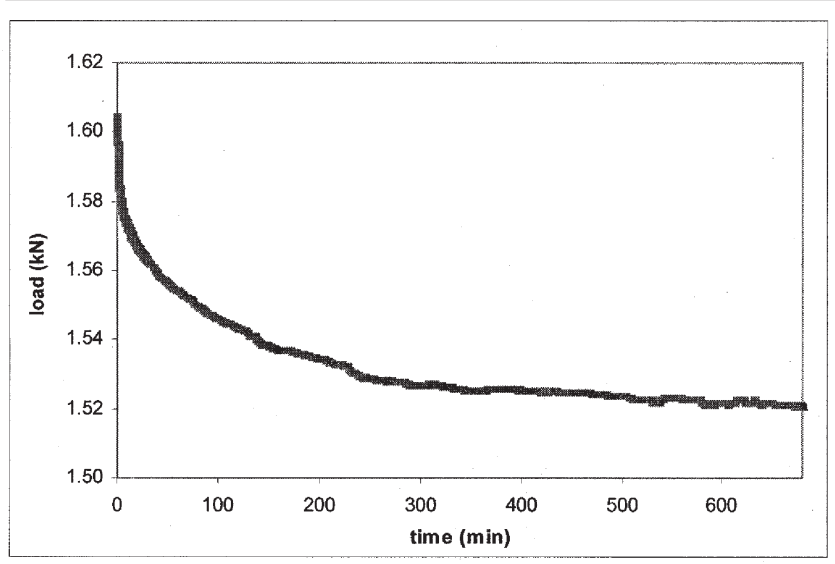

Fig. 10. Stabilization in the load during a 14-h stress relaxation test at 0.75 strain amplitude for $455-\mu \mathrm{m}$ sample.

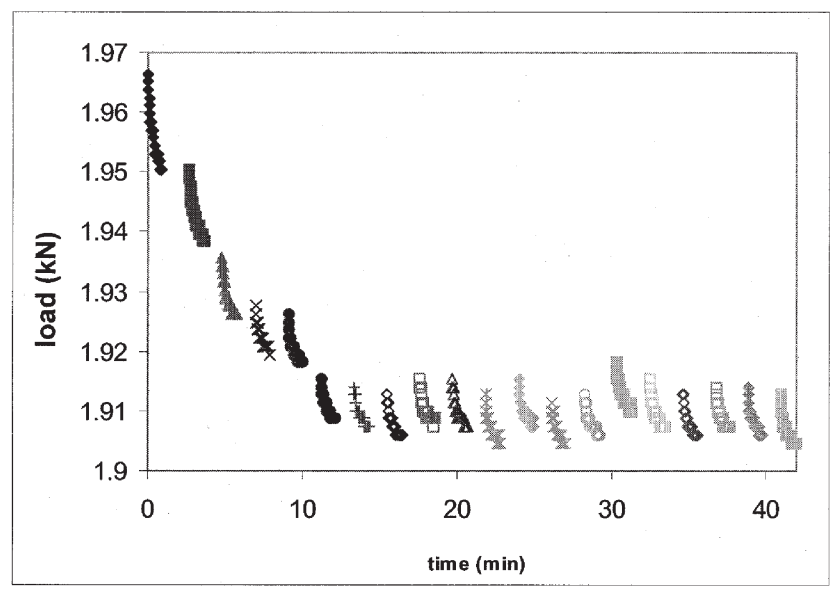

Fig. 11. Stress relaxation data for 20 cycles for the $509-\mu \mathrm{m}$ sample cycled to 0.75 strain and held for $1 \mathrm{~min}$.

stress relaxation decreases much more rapidly (10 min versus 5 h) with cycling. Each application of reversed stress contributes to microstructural damage, and therefore, the decrease in load is achieved sooner than a single cycle with long hold times. Further analysis of the effect of reverse stress on the stress-relaxation behavior is presented later in this paper.

The samples in Fig. 12 had a hold time of 5 min at the maximum strain amplitude. These samples experienced significantly higher loads and displayed load drops to roughly $75 \%$ of the original load after 15-20 cycles. The plots also suggest that the loads will continue to decrease upon further cycling. The large loads applied to these samples also resulted in visible damage on the surface of the solders (Table I). Significant stress relaxation (or drops in the load during holds at the maximum strain) can be correlated with large applied loads and substantial surface damage.

During the stress-relaxation period, the solder microstructure may be readjusting to handle the imposed load. Microcracks or fracture would result in a decrease in load-bearing ability and would account for the decrease in load per cycle for a constant strain amplitude.

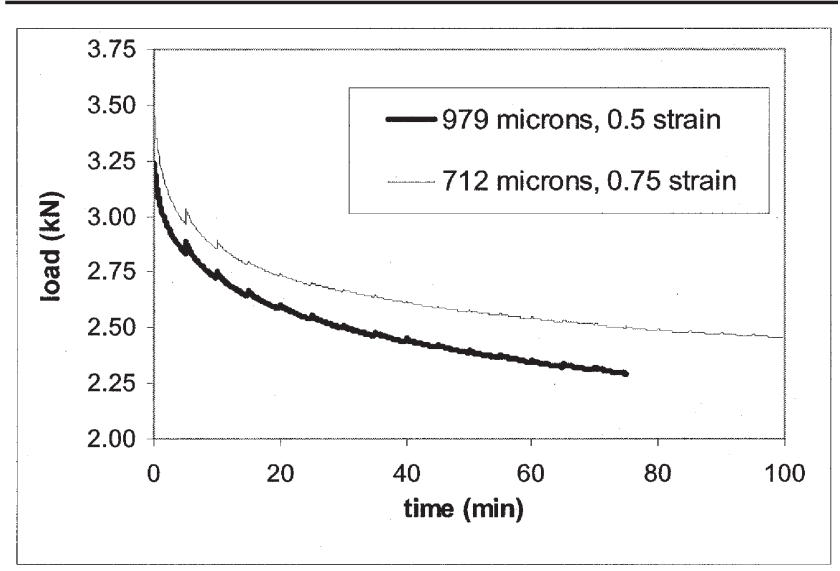

Fig. 12. Large load drops during stress relaxation ( $5 \mathrm{~min}$ ) and between cycles for samples that experience large applied loads. No plateau in load is achieved after 15-20 cycles. 
The mechanical behavior of the solders appears to be most affected by the imposed load, rather than the joint thickness or applied strain amplitude. Figure 13 plots the peak load per cycle for several samples of different joint thickness and of different strain amplitudes. When comparing samples of the same strain amplitudes, the thicker joint samples experienced larger load drops during hold times and between cycles. However, to achieve the same strain amplitudes, larger applied loads were required for the thicker joints. Likewise, samples of similar thickness values with different applied strain amplitudes were subjected to different loads. The larger applied strain (and, thus, load required) resulted in larger load drops during hold times and between cycles.

The significance of the effect of the load on solders may still have to be related to the strain amplitude (or changes in temperature) of the particular application. In realistic settings, the load is not really controlled but perhaps can be adjusted through the joint thickness or size.

To obtain a better understanding of the processes involved during repeated reverse straining, stressstrain plots were obtained for each straining cycle. These plots are provided in Figs. 14-16. These plots

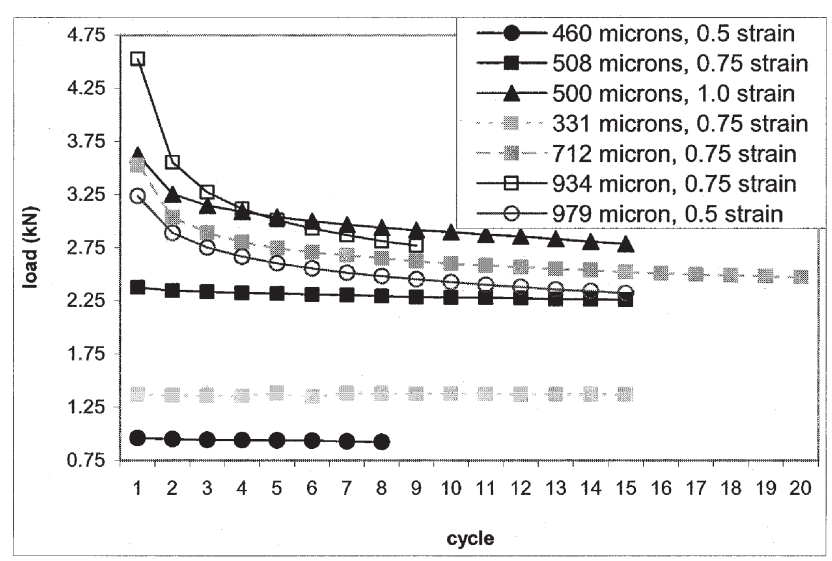

Fig. 13. The peak load per cycle for several samples. Samples that experience large loads show the largest load drops per cycle.

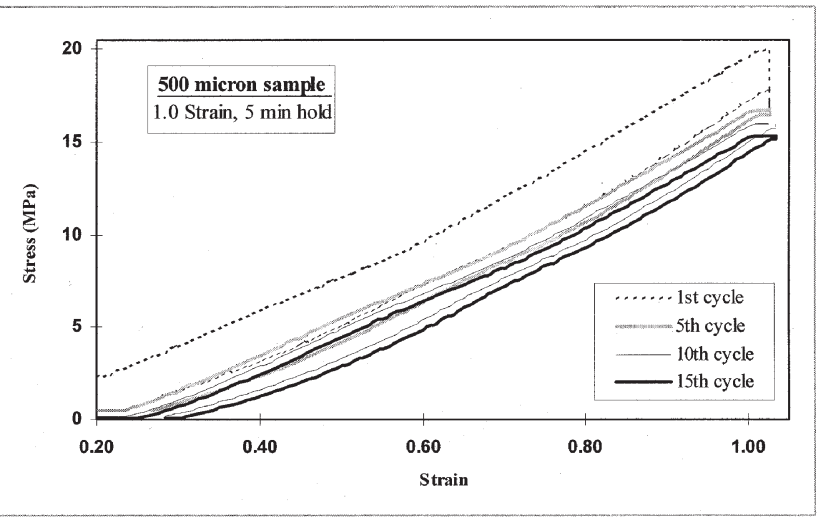

Fig. 14. Various loading and unloading cycles for the $500-\mu \mathrm{m}$ sample tested to 1.0 strain. The stress decreases with successive cycles. The stress drop during the hold time at the maximum strain is the largest for the first cycle.

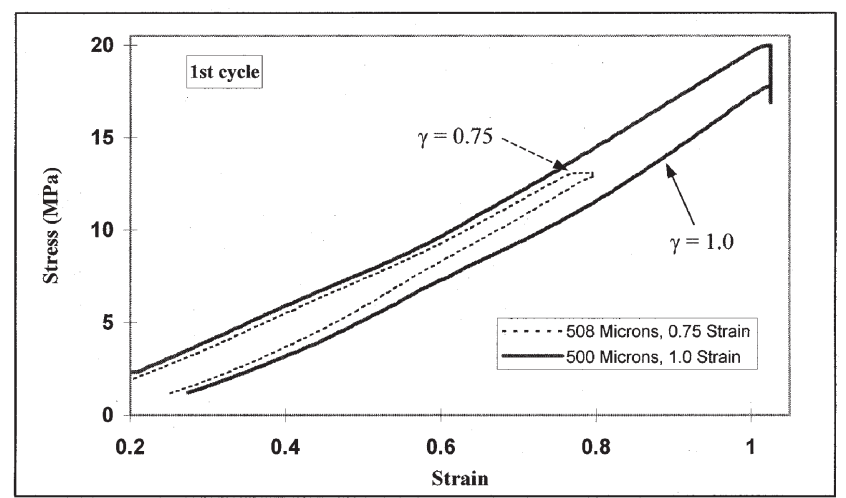

Fig. 15. Stress-strain curves for the first cycle for samples of similar joint thickness at different strain amplitudes. Larger strain amplitudes require large stresses.

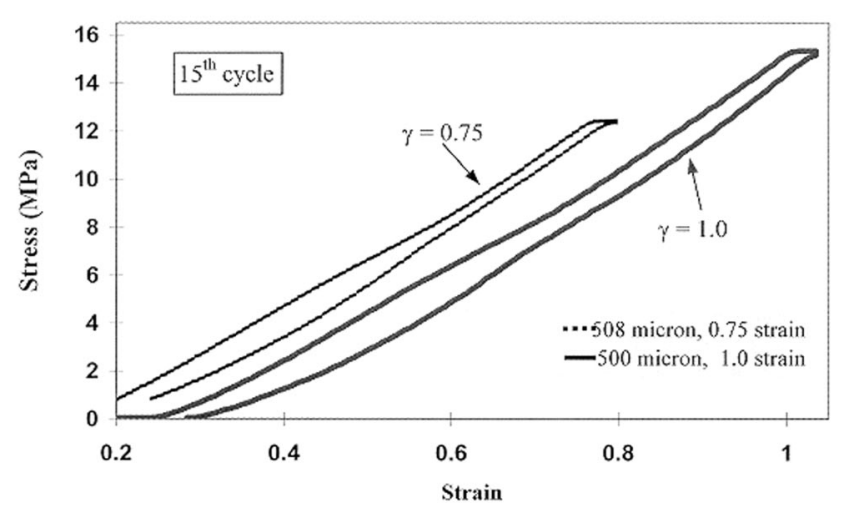

Fig. 16. Stress-strain curve of the 15th cycle for samples of similar joint thickness at different strain amplitudes. The sample with larger strain amplitude has decreased its load bearing ability.

incorporate the change in stress under constant strain that occurs during relaxation (i.e., hold times) at the maximum strain portion of the cycle. No attempts were made to incorporate the relaxation data at the low strain extreme. Figure 14 compares the loading and unloading portions as a function of number of cycles. With an increasing number of cycles, the difference between the loading and unloading curves tends to decrease and ultimately disappears after several cycles. The same trend is consistent with Figs. 9 and 11.

Figure 15 illustrates the stress-strain behavior of two specimens with solder-joint thickness about 500 $\mu \mathrm{m}$ for 0.75 and 1 shear-strain amplitudes during the first cycle. As anticipated, the higher strain amplitude warranted higher stress. The important feature is that the specimen that experiences the higher strain amplitude also shows more significant difference between the loading and unloading parts as compared to the one that experiences lower strain amplitude. Figure 16 compares the stressstrain plots for the 15th loading cycle for 0.75 and 1 shear amplitudes for the same two specimens in Fig. 15. Interestingly, the specimen that undergoes lower strain amplitude per cycle requires higher stress for the same strain as compared to that for a specimen that undergoes larger strain amplitude per cycle. 
These plots tend to suggest that during the early parts of repeated reverse straining, microcracks develop. The number of cracks increase with further cycling but do not connect together to cause failure. Such events will decrease the maximum load experienced during successive cycles. Also the energy absorbed during successive cycles will tend to decrease because less and less number of cracks will be needed to accommodate the strain. Crack opening and closing can accommodate the imposed strain. Such a scenario may also explain the residual strength of TMF solder joints, where a significant decrease in strength occurs during the first few hundred cycles. It appears that crack propagation, which requires the joining of these microcracks, may be the controlling mechanism of failure in these cases.

To relate results from this study with the creep behavior of other Sn-Ag eutectic solders, ${ }^{12,17}$ analysis of the load drops associated with the reverse stressing was carried out with conventional stress relaxation using Eq. 1: ${ }^{12}$

$$
\dot{\gamma}=\mathrm{A} \tau^{\mathrm{n}} \exp (-\mathrm{Q} / \mathrm{kT})
$$

where $\dot{\gamma}$ is the shear-strain rate, A is the pre-exponential constant, $\tau$ is the shear stress, $\mathrm{n}$ is the stress exponent, $\mathrm{Q}$ is the activation energy, $\mathrm{k}$ is the Boltzmann's constant, and $\mathrm{T}$ is the absolute temperature. The shear-strain rate is related to the change in load per unit time $(\dot{\mathrm{P}})$ via

$$
\dot{\gamma}=\frac{-\dot{\mathrm{P}}}{\mathrm{Kh}}
$$

where $\mathrm{K}$ is the combined stiffness of the machine and the specimen, and $\mathrm{h}$ is the thickness of the solder joint. $^{12}$

Figure 17 displays the shear-strain rate versus the shear stress on a log-log scale. Stress relaxation occurs during the hold times of successive cycles and is represented by the open data points. In addition, data from a sample experiencing uninterrupted stress relaxation and from a single shear lap creep specimen are plotted in the same figure. Larger applied stresses result in more stress relaxation, as shown in Eq. 1. Similarly, larger load drops were associated with larger applied loads (Fig. 13). The rate of stress relaxation decreases significantly as the stress drops.

The stress exponents for several samples under repeated loading fall within the range of 9.2-12.2 and are comparable to the value $(\mathrm{n}=13.7)$ obtained for the same solder under standard stress-relaxation conditions (i.e., single cycle and long hold time). Furthermore, these stress exponent values are very close to values quoted in the literature for the same solder. ${ }^{12,17}$ The data for the single shear lap creep specimen in Fig. 17 gives a stress exponent of 11.

While the stress exponents are very similar, the actual strain-rate values differ dramatically for the samples under repeated loading conditions. For the same stress, significantly higher strain rates are ex-

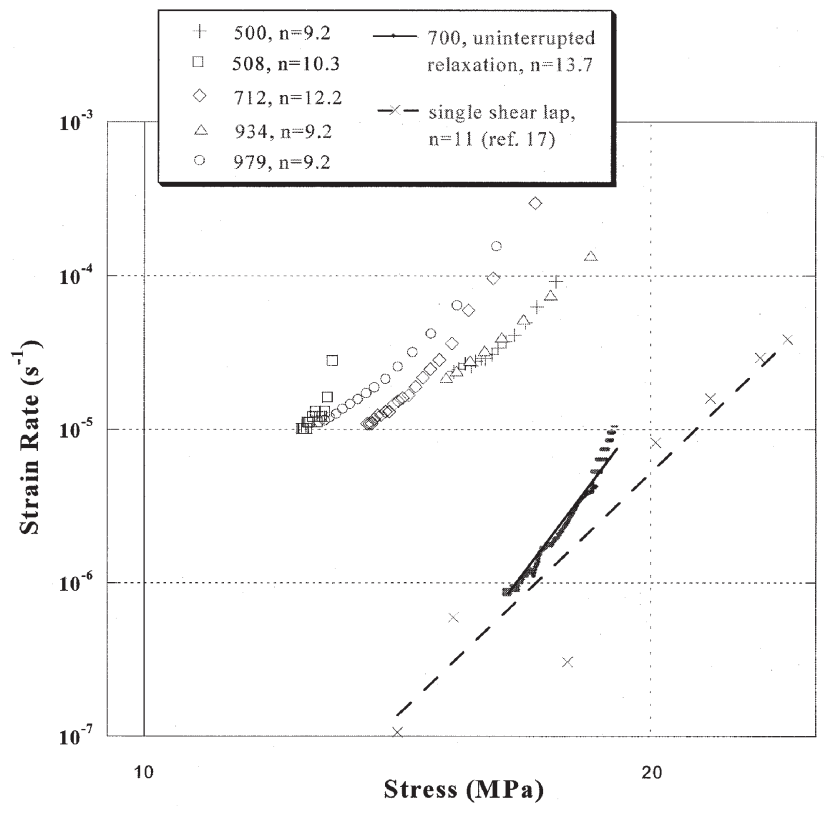

Fig. 17. Steady-state strain rate versus stress of Sn-Ag eutectic solder joints during relaxation for cycled (reverse sheared) samples, a single-cycle-long hold sample, and a single shear lap creep specimen. The stress exponent values all fall within the range of 9.213.7; however, the strain rate values for a particular stress are much higher for the reversed sheared samples.

perienced when the samples are subjected to cycling under large loads. This result may be attributed to the accelerated damage accumulation that takes place under repeated reverse stressing.

\section{CONCLUSIONS}

A new testing method was developed to study the effects of reverse shear on $\mathrm{Sn}-\mathrm{Ag}$ eutectic solders under constrained conditions. When cycled between a maximum- and minimum-strain amplitude, samples were subjected to reversed shear-stress states. Damage accumulation on the surface correlated with the observed mechanical behavior. The following observations were made.

- Upon loading, striations and deformation bandings occurred along the Sn dendrites. Continued cycling of reverse shearing resulted in additional damage accumulation with the striations becoming more intense.

- A threshold in load $(\sim 2.5 \mathrm{kN})$ was needed for any visible damage on the surface of the solder samples. The load-threshold value relates very closely to the yield stress of Sn.

- Hold times at the maximum strain amplitude (i.e., stress-relaxation studies) showed that full relaxation to zero load is not achieved with the constrained samples that contained two $3 / 8$ in. $\times 3 / 8$ in. $\times 1$ in. soldered areas. Stress relaxation decreases with each successive cycle.

- For a constant applied strain amplitude, the maximum load (and stress) decreases with each successive cycle. Microcracks may be developing during the repeated reverse shear. 
- Stress exponent values obtained from $\log \dot{\gamma}$ versus $\log \tau$ plots during stress-relaxation segments under repeated reverse stressing were comparable to those obtained during stress relaxation after monotonic loading and creep experiments. However, the strain-rate values for a particular stress were much higher for the reverse stressed samples.

\section{ACKNOWLEDGEMENTS}

The authors thank the National Science Foundation for providing additional financial support as a Research Opportunity Award (ROA) to project Contract No. NSF-DMR-0081796. The authors also thank Hong Joo Rhee and Hairong Jiang for their help during the preparation of this manuscript.

\section{REFERENCES}

1. A.W. Gibson, K.N. Subramanian, and T.R. Bieler, J. Adv. Mater. 30, 19 (1998).

2. S. Choi, T.R. Bieler, J.P. Lucas, and K.N. Subramanian, J. Electron. Mater. 28, 1208 (1999).

3. F. Guo, J. Lee, S. Choi, J.P. Lucas, T.R. Bieler, and K.N. Subramanian, J. Electron. Mater. 30, 1073 (2001).

4. K.N. Tu, T.Y. Lee, J.W. Jang, L. Li, D.R. Frear, K. Zeng, and J.K. Kivilahti, J. Appl. Phys. 89, 4843 (2001).

5. K.N. Tu and K. Zeng, Mater. Sci. Eng. 34, 1 (2001).

6. H. Conrad, Z. Guo, Y. Fahmy, and D. Yang, J. Electron. Mater. 28, 1062 (1999).

7. A.U. Telang, S. Choi, T.R. Bieler, and K.N. Subramanian, J. Mater. Research, 17 (9), 2294 (2002).

8. S. Choi, A.W. Gibson, J.L. McDougall, T.R. Bieler, and K.N. Subramanian, in Design and Reliability of Solders and Solder Interconnections, eds. R.K. Mahidhara, S.M.L. Sastry, and P.K. Liaw (Warrendale, PA: TMS, 1997), pp. 241-245.
9. A.W. Gibson, S. Choi, K.N. Subramanian, and T.R. Bieler, Design and Reliability of Solders and Solder Interconnections, eds. R.K. Mahidhara, S.M.L. Sastry, and P.K. Liaw (Warrendale, PA: TMS, 1997), pp. 97-103.

10. H.L. Reynolds, S.H. Kang, and J.W. Morris, Jr., J. Electron. Mater. 28, 69 (1999)

11. R. Darveaux, K.L. Murty, and J. Turlik, JOM, 44 (7), 36 (1992).

12. S.G. Jadhav, K.N. Subramanian, T.R. Bieler, and J.P. Lucas, J. Electron. Mater. 30, 1197 (2001).

13. S. Choi, K.N. Subramanian, J.P. Lucas, and T.R. Bieler, J. Electron. Mater. 29, 1249 (2000)

14. C.H. Reader, R.W. Messler, and L.F. Coffin, J. Electron. Mater. 28, 1045 (1999).

15. Y.H. Pao, S. Badgley, R. Govila, and E. Jih, Fatigue of Electronic Materials, eds. S.A. Schroeder and M.R. Mitchell (Philadelphia, PA: ASTM, 1994), pp. 60-81.

16. J. Sigelko, S. Choi, K.N. Subramanian, and J.P. Lucas, J. Electron. Mater. 29, 1307 (2000).

17. S. Choi, J. Lee, F. Guo, T.R. Bieler, K.N. Subramanian, and J.P. Lucas, JOM 53, 22 (2001).

18. F. Guo, J. Lee, J.P. Lucas, K.N. Subramanian, and T.R. Bieler, J. Electron. Mater. 30, 1222 (2001).

19. F. Guo, J.P. Lucas, and K.N. Subramanian, Solder. Surf. Mount Technol. 13, 7 (2001).

20. F. Guo, J.P. Lucas, and K.N. Subramanian, J. Mater. Sci.: Mater. Electron. 12, 27 (2001).

21. S. Ahat, M. Sheng, and L. Luo, J. Electron. Mater. 30, 1317 (2001)

22. S. Choi, J.G. Lee, K.N. Subramanian, J.P. Lucas, and T.R Bieler, J. Electron. Mater. 31, 292 (2002).

23. J.G. Lee, F. Guo, S. Choi, K.N. Subramanian, T.R. Bieler, and J.P. Lucas, J. Electron. Mater., 31 (9), 946 (2002),

24. J. Howell, A. Telang, J.G. Lee, K.N. Subramanian, J. Mater. Sci.: Mater. Electron., 13 (6), 335 (2002).

25. C. Lonskey, private communication (2002) 